\title{
Single-slit split-ring resonators at optical frequencies: limits of size scaling
}

\author{
M. W. Klein, C. Enkrich, and M. Wegener \\ Institut für Angewandte Physik and DFG-Center for Functional Nanostructures (CFN), Universität Karlsruhe (TH), \\ Wolfgang-Gaede-Straße 1, D-76131 Karlsruhe, Germany \\ C. M. Soukoulis \\ Ames Laboratory and Department of Physics and Astronomy, Iowa State University, Ames, Iowa 50011
}

\section{S. Linden}

Institut für Nanotechnologie, Forschungszentrum Karlsruhe in der Helmholtz-Gemeinschaft, Postfach 3640, D-76021 Karlsruhe, Germany

\begin{abstract}
Received January 3, 2006; accepted January 30, 2006; posted February 9, 2006 (Doc. ID 66981)
We present magnetic metamaterials composed of $35 \mathrm{~nm}$ minimum feature-size gold split-ring resonators with a fundamental magnetic resonance at a wavelength of $900 \mathrm{~nm}$. Corresponding calculations reveal excellent agreement with the experiments and show that the limits of size scaling have been reached. (C) 2006 Optical Society of America

OCIS codes: $160.4760,260.5740$.
\end{abstract}

Split-ring resonators ${ }^{1}$ (SRRs) allow for artificial magnetism at elevated frequencies and have recently attracted considerable attention. ${ }^{2-7}$ In essence, a SRR is just a small $L C$ circuit consisting of an inductance $L$ and a capacitance $C{ }^{5}$ Dense packing of SRRs, using lattice constants smaller than the $L C$ resonance wavelength, leads to effective materials that can exhibit a resonance behavior of the magnetic permeability $\mu$, with a magnetic resonance at the $L C$ frequency $\omega_{L C}=1 / \sqrt{L C}$. The simplest form is one metallic ring with one slit. ${ }^{1}$ The ring forms one winding of a coil (the inductance), and the ends of the ring form the plates of a capacitance. Theory has shown several times that both the inductance and the capacitance scale proportionally to SRR size, provided that all SRR dimensions are scaled down simultaneously. ${ }^{8,9}$ Thus the $L C$ frequency scales inversely with normalized size (or scale factor) $s$, i.e., $\omega_{L C} \propto 1 / s$. This scaling law, however, holds only as long as the metal acts as an ideal metal. It is clear that the limit is determined by the metal plasma frequency. Recent theoretical work has addressed this aspect in more detail. ${ }^{10,11}$

It is our aim in this Letter to present what we believe to be the first experiments in a regime where the SRR size scaling breaks down, i.e., at the longwavelength end of the visible range. In this regime, the Drude model of metals (that has been used extensively for describing SRRs) becomes questionable. Thus we compare our experiments with calculations that use the complex dielectric function of the metal as determined from experiments in the literature.

Before we address our experiments, we briefly recall an intuitive reasoning for the end of SRR size scaling ${ }^{10}$ : to support a current $I$ in the coil, one needs the magnetic energy $\frac{1}{2} L I^{2}$. In addition, the kinetic energy of the charged carriers has to be provided. For an ideal metal with infinite carrier density, hence in- finite plasma frequency, the carrier velocity and the kinetic energy are zero, even for finite current $I$. For a real metal, velocity and kinetic energy become finite. As velocity and current are proportional, the electron kinetic energy can be recast into an effective additional magnetic energy with a "kinetic" inductance $L_{\text {kin }}$, which adds to the usual inductance, i.e. $L \rightarrow L+L_{\text {kin }}$, and which scales inversely with size. ${ }^{10}$ Thus it is negligible for large inductances but becomes dominant for small SRRs. In this limit the $L C$ frequency approaches a constant, $\omega_{L C}^{\max }$; i.e., it becomes independent of the SRR size if all SRR dimensions are scaled by the same scale factor $s$. This reasoning altogether leads to the modified scaling law ${ }^{10}$

$$
\omega_{L C} \propto \frac{1}{\sqrt{s^{2}+\text { const. }} .}
$$

It should be clear that the $L C$ frequency could be further increased by reducing the net capacitance $C$, which is possible, for example, by introducing further slits into the ring. ${ }^{10}$ Here we focus on single-slit SRRs because they exhibit the largest ratio of resonance wavelength and SRR size, i.e., they are ideal for constructing effective materials (the true metamaterial limit).

In our experiments, we continue along the lines of our previous work that started ${ }^{5}$ with single-slit SRRs at a resonance wavelength of $3 \mu \mathrm{m}$ and recently ${ }^{6}$ arrived at a resonance wavelength of $1.5 \mu \mathrm{m}$ (and shorter-wavelength higher-order magnetic resonances ${ }^{6}$ ). Again, the samples are fabricated by using standard electron-beam lithography and electron-beam evaporation ${ }^{6}$ and consist of gold SRRs on a glass substrate. To avoid charging effects in the electron-beam fabrication process, the substrates are coated with a $5 \mathrm{~nm}$ thin indium-tin-oxide (ITO) layer. Electron micrographs of a typical structure with a footprint of $(20 \mu \mathrm{m})^{2}$ and a gap width of $35 \mathrm{~nm}$ 


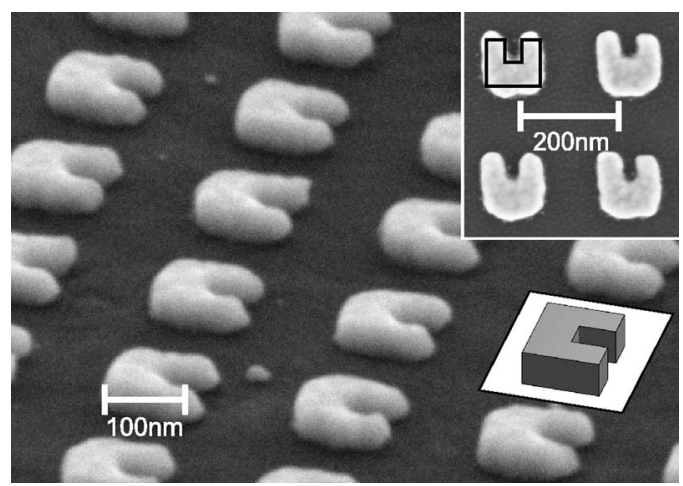

Fig. 1. Oblique-view electron micrograph of the gold SRR array. The upper inset shows a top-view image, and the black line depicts the lateral dimensions of the SRR used in the calculations. The lower inset is a corresponding perspective view, shown on the same scale as the large micrograph.

are shown in Fig. 1. To enhance the magnetic response, we have not scaled down the gold film thickness but have rather increased it with respect to our previous work. ${ }^{5,6}$ As a result, the SRRs are no longer flat objects but instead reveal a significant aspect ratio of height to lateral dimensions, as apparent from Fig. 1.

Optical transmittance spectra for linear incident polarization are measured with a homebuilt setup, allowing for investigating small samples at a small opening angle $\left(5^{\circ}\right)$ of the incident light to avoid angleaveraging effects as much as possible. Closely similar to our previous work on larger SRRs, ${ }^{5,6}$ we find two resonances when exciting the structures with horizontal polarization [see Fig. 2(a)]: the $900 \mathrm{~nm}$ wavelength resonance is the magnetic resonance excited by the electric-field component of the incident light. It corresponds to a ring current in the SRR leading to a magnetic dipole moment perpendicular to the SRR plane. The short-wavelength resonance is the usual Mie resonance corresponding to an electric dipole oscillating in the horizontal bottom SRR arm. For vertical incident polarization [see Fig. 2(b)], only one Mie resonance is observed, corresponding to electric dipoles oscillating in the two vertical SRR arms. We have recently shown that the relative positions of the magnetic resonance and the Mie resonance sensitively depend on the relative depth of the SRR opening. ${ }^{7}$ Thus it is not easy to investigate the SRR size scaling experimentally, since the condition that all SRR dimensions be scaled by the identical factor is difficult to fulfill.

Thus we follow a different route here. We first compare our experimental results with a refined theoretical treatment, leading to almost quantitative agreement. In other words, our SRRs come close to the theoretical ideal. Next, we perform numerical size scaling "experiments." Previous theoretical work has employed the Drude model to describe the metal dielectric function. ${ }^{10,11}$ It is well known, however, that all metals exhibit (significant) deviations with respect to the Drude model when approaching very large frequencies. Thus we use the measured complex dielectric function of gold, which is well docu- mented in the form of tables in the literature ${ }^{12}$ for frequencies between 155 and $1595 \mathrm{THz}$ (for frequencies below $155 \mathrm{THz}$, we have to use the Drude model). Those measurements have been performed on gold films of comparable thickness. Our numerical simulations employ the FEMLAB software package, which is based on a finite-element method and used in the frequency domain with vector elements and periodic boundary conditions. The parameters are $l_{x}$ $=110 \mathrm{~nm}, l_{y}=95 \mathrm{~nm}, w_{x}=38 \mathrm{~nm}, w_{y}=50 \mathrm{~nm}$, thickness $t=45 \mathrm{~nm}$, and a square lattice with lattice constant $a=240 \mathrm{~nm}$ [see the inset in Fig. 2(d)]. This geometry is a fair approximation of the experimental SRR structures (see Fig. 1), which are rounded off by the fabrication process. The substrate refractive index is 1.5. The corresponding calculations are depicted in the second row of Fig. 2. The bare calculations agree well regarding the spectral positions of the resonances; however, the calculated resonances are somewhat deeper and narrower than in the experiment. From the electron micrographs shown in Fig. 1 it is clear that the SRR dimensions vary slightly throughout the array, which translates into a certain inhomogeneous broadening. This aspect can be described by convolving the bare response with a normalized Gaussian distribution (in the frequency domain). Choosing a half-width at half-maximum (HWHM) corresponding to $3 \%$ of the center frequency, the result agrees very well with our experiments [see Fig. 2(a)].

Are our experiments within or outside the limit of SRR size scaling? To investigate this question, we repeated the calculations (with the actual gold dielectric function as above) for two cases: (i) All SRR dimensions are scaled simultaneously by factor $s$ and (ii) only the lateral SRR dimensions are scaled by $s$

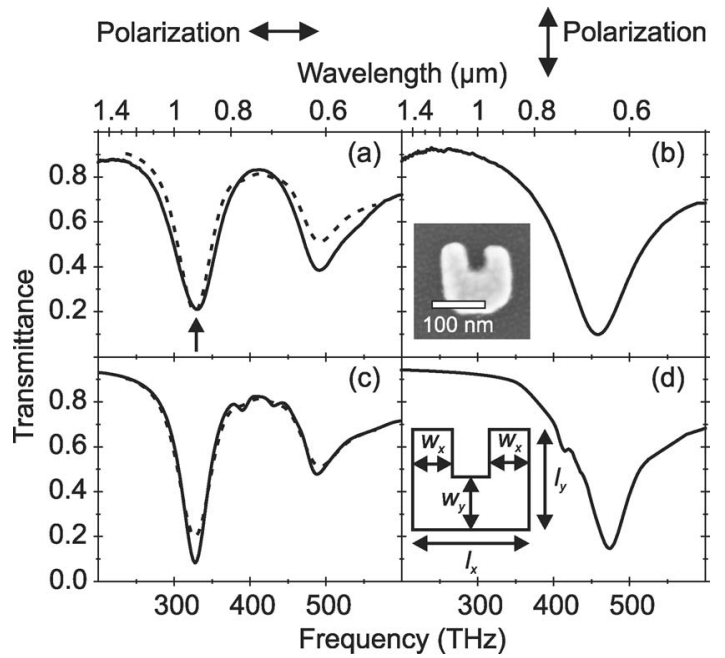

Fig. 2. First row: experimental transmittance spectra for (a) horizontal and (b) vertical incident polarization (see arrows). The inset shows a top-view electron micrograph of a gold SRR. Second row: (c) and (d) exhibit corresponding calculated spectra (solid curves). To mimic the influence of inhomogeneous broadening, the dashed curve depicted in both (a) and (c) is the convolution of the solid curve in (c) with a Gaussian distribution $(\mathrm{HWHM}=3 \%$ of the center frequency). The magnetic resonance frequency (see Fig. 3) is indicated by an arrow in (a). 


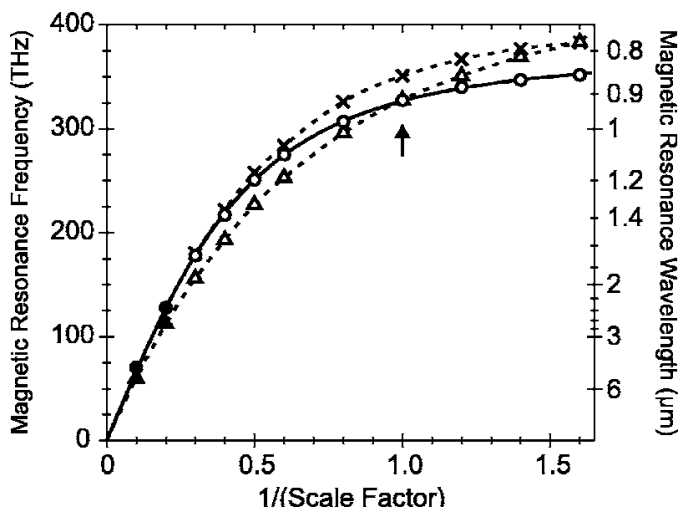

Fig. 3. Scaling behavior of the magnetic resonance frequency [compare arrow in Fig. 2(a)]. The SRR size is referenced with respect to the parameters of Fig. 2 (see arrow here). (i) For the circles, all geometrical parameters and the lattice constant are scaled by the same scale factor $s$. (ii) For the triangles, only the lateral parameters are scaled, the thickness is fixed. For the open symbols, the measured metal permittivity of gold is used in the calculations, while for the full symbols a Drude permittivity for gold is used. The solid curve corresponds to a fit using the simple for-

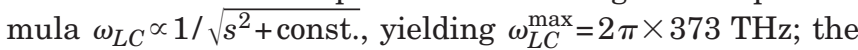
dashed curves are guides to the eye. The crosses are for (i), but for silver rather than for gold.

(i.e., the gold thickness remains fixed). The results are depicted in Fig. 3, where we plot the magnetic resonance frequency (compare the arrow in Fig. 2) versus the inverse scale factor $s$. For both cases, (i) and (ii), the experiment is already outside the scaling regime $\omega_{L C} \propto 1 / s$. Thus it is no longer expected that the magnetic permeability $\mu$ becomes negative close to resonance. ${ }^{10}$ Note that the simple formula (1) describes the numerical results for case (i) extremely well.

Silver is often considered to be close to an ideal metal at optical frequencies. Thus we have repeated the calculations, replacing the gold with silver. The complex permittivity of silver is again taken from Ref. 12. The results for scaling all the SRR parameters (the crosses in Fig. 3) are very closely similar to those for gold (the circles in Fig. 3). For the parameters investigated, the maximum deviation in magnetic resonance frequency is less than $10 \%$ toward higher frequencies, consistent with a corresponding difference in the plasma frequencies of gold and silver. However, it is well known that silver rapidly oxidizes unless protective measures are taken. The SRRs could, for example, be coated with a dielectric film, which, unfortunately, would again redshift the resonances.

In conclusion, we have fabricated metamaterials consisting of dense arrays of (single-slit) split-ring resonators with a fundamental magnetic resonance at a wavelength of about $900 \mathrm{~nm}$. The measured transmittance spectra agree very well with the calculations, revealing that these SRR structures are of high quality and that they are already outside the size-scaling regime, i.e., further miniaturization would not significantly increase the magnetic resonance frequency.

We acknowledge support by the Deutsche Forschungsgemeinschaft (DFG) and the State of Baden-Württemberg through the DFG-Center for Functional Nanostructures (CFN) within subproject A1.5. M. Wegener further acknowledges support under project DFG-We 1497/9-1. C. M. Soukoulis acknowledges support by the Alexander von Humboldt Senior-Scientist Award 2002 and by Ames Laboratory (contract W-7405-Eng-82) and the EU FET project DALHM METAMORHOSE, and PHOREMOST and DARPA (contract HR0011-05-C-0068). S. Linden acknowledges support through a Helmholtz-HochschulNachwuchsgruppe (project VH-NG-232). M. W. Klein's e-mail address is matthias.klein@physik.unikarlsruhe.de. C. M. Soukoulis is also with the Institute of Electronic Structure and Laser at the Foundation for Research and Technology-Hellas (FORTH), and the Department of Materials Science and Technology, University of Crete, Heraklion, Crete, Greece.

\section{References}

1. J. B. Pendry, A. J. Holden, D. J. Robbins, and W. J. Stewart, IEEE Trans. Microwave Theory Tech. 47, 2075 (1999).

2. R. A. Shelby, D. R. Smith, and S. Schultz, Science 292, 77 (2001)

3. T. J. Yen, W. J. Padilla, N. Fang, D. C. Vier, D. R. Smith, J. B. Pendry, D. N. Basov, and X. Zhang, Science 303, 1494 (2004).

4. D. R. Smith, J. B. Pendry, and M. C. K. Wiltshire, Science 305, 788 (2004).

5. S. Linden, C. Enkrich, M. Wegener, J. F. Zhou, T. Koschny, and C. M. Soukoulis, Science 306, 1351 (2004).

6. C. Enkrich, M. Wegener, S. Linden, S. Burger, L. Zschiedrich, F. Schmidt, J. F. Zhou, T. Koschny, and C. M. Soukoulis, Phys. Rev. Lett. 95, 203901 (2005).

7. C. Enkrich, F. Pérez-Willard, D. Gerthsen, J. F. Zhou, C. M. Soukoulis, M. Wegener, and S. Linden, Adv. Mater. 17, 2547 (2005).

8. S. O'Brien and J. B. Pendry, J. Phys. Condens. Matter 14, 6383 (2002).

9. S. O’Brien, D. McPeake, S. A. Ramakrishna, and J. B. Pendry, Phys. Rev. B 69, R241101 (2004).

10. J. F. Zhou, T. Koschny, M. Kafesaki, E. N. Economou, J. B. Pendry, and C. M. Soukoulis, Phys. Rev. Lett. 95, 223902 (2005).

11. A. Ishikawa, T. Tanaka, and S. Kawata, Phys. Rev. Lett. 95, 237401 (2005).

12. P. B. Johnson and R. W. Christy, Phys. Rev. B 6, 4370 (1972). 